\title{
High-performance materials for biomedical applications-a short review
}

\begin{abstract}
The present review articulates some the properties of high-performance materials such as carbon/glass reinforced composite (resin-based or otherwise) that make these materials a highly potent substitute for many usual implants and assistive devices. The mechanical aspects such as high strength-to-weight ratio and favorable thermal as well as thermos-mechanical properties make such materials extremely desirable for biomedical applications. Based on these properties and available literature the various uses of these high-performance bio-materials are advocated in this review.
\end{abstract}

Keywords: implants, prosthetic socket, mechanical, biomedical, materials
Volume I Issue 5 - 2017

\author{
Shreeshan Jena,' Subrata Kumar Panda, ${ }^{2}$ \\ Arunachalam Thirugnanam' \\ 'Department of Biotechnology and Medical Engineering, \\ National Institute of Technology Rourkela, India \\ ${ }^{2}$ Department of Mechanical Engineering, National Institute of \\ Technology Rourkela, India
}

Correspondence: Subrata Kumar Panda, Department of Mechanical Engineering, National Institute of Technology Rourkela, Rourkela, Odisha, India-769008, India, Tel +9| 66I 246 2529, Email pandask@nitrkl.ac.in

Received: October 28, 2017 | Published: December 08, 2017

\section{Introduction}

Owing to the advances in the high-performance materials create tremendous scope for their possible applications in a variety of fields including but not limited to the industries like mechanical, aerospace, electronics, textile, military and biomedical (orthodontics, prosthetics, orthotics, orthopedic implants, etc.). The need of the hour is to improvise upon and develop materials that may establish their usefulness in the multi-disciplinary applications. The resin based composite materials have been studied with various reinforcing materials (glass/carbon/carbon nanotubes and organic fibers, etc.) and the analyses are largely limited to the strength and tribological aspects. Out of all composites, few materials are perfectly biocompatible for the application with the human body and their high tensile strength, light weight, low cost and ease of fabrication make them highly desirable candidates for biomedical uses for treatment as well as rehabilitation purposes. While the mechanical behaviour of these materials has been explored sufficiently, the interaction with biological medium, i.e. behaviour within the human body has not been studied greatly. Besides, some composites have quite good thermal properties that make them suitable for applications in the design of High-performance materials for biomedical applications prosthetic sockets to prevent ulceration as well as for preparing tactile clothing that can provide good comfort to the user.

Thus, the present work proposal revolves around the applications of high-performance polymer composites in the fields of rehabilitation and biomedical engineering. These materials have huge scope for developing high-strength, light weight and comfortable composites in rehabilitation engineering as compared to the presently available materials. The mechanical as well as biological interaction of these materials shall be simulated using numerical methods for the analysis of non-linear materials. The effect of the thermal conductivity of these composites can also be studied experimentally as well as through finite element simulations to determine their suitability as fabrics that present good comfort in harsh environments as well as for in the inner socket linings of prosthetics. This project shall also lay focus on improving the thermal efficiency of carbon nanotubes and other composite combinations for increasing the range of possible applications.

\section{Discussion}

The use of synthetic biomaterials in regenerative medicine and rehabilitation has been prevailing for more than four decades. Some of the various biomaterials that have been used till date in orthopedic applications are ceramics, metals and alloys, polymeric biomaterials and composite biomaterials. One of the most commonly encountered biomaterial is bone cement or poly-methyl-methacrylate (PMMA) that is used in a variety of orthopedic treatments and surgeries. This bone cement, that provides a bond between the implant and the bone tissue, may also be reinforced by carbon fibers to provide an even longer term of stability of the fixation. ${ }^{1}$ However, this cement is usually never used by itself and almost every time accompanies a metallic, ceramic or polymer implant. The complex bone geometry of the human body and the treatment modalities often require the fabrication of implants in varied shapes and sizes. Thus, these implants have to undergo a plethora of machining and treatment steps before they are available to the surgeon and hence the high cost.

The need for making use of composite biomaterials as a feasible and preferable alternative in comparison to metallic implants is what has led researchers to study the properties of these materials. Besides possessing high tensile strength and a density close to that of the human bone, these composites are easy to fabricate in different shapes at a low cost. ${ }^{2}$ Further, there are multiple studies that explore the use of carbon fiber composites to serve as replacement for metallic implants. ${ }^{3-5}$ The biopolymers, dematerialized bone matrices and natural fibers also provide an innate increased resistance to corrosion in the human body environment, owing to which the implants fabricated of these materials shall present a more conducive environment for bone growth during healing. ${ }^{6,7}$

The use of these composites is not limited to just implant applications, and extends to external fixations and prosthetic applications such as prosthetic sockets, liners, foot, fabric resistance temperature detectors (for supracutaneous applications, smart 
clothing, medical electronics, etc.). ${ }^{8-11}$ The non-functionalized carbon nanotubes may also be used to augment poly-methyl-methacrylate (PMMA) matrix for cementing prosthetics, medical implants, dental restorations or fixations. ${ }^{12}$ The composites available presently do not have sufficiently high thermal properties and multiple researches have been reported that have aimed at enhancing the thermal properties with the addition of single walled carbon nanotubes (SWCNTs) ${ }^{9-18}$

\section{Conclusion}

The review of the potential applications of the high-performance materials for the biomedical industry clearly indicates that there is more than sufficient scope for these materials due to their unmatched properties. In view of this, it can be said that these smart materials may be the future of the rehabilitation industry.

\section{Acknowledgements}

None.

\section{Conflict of interest}

The authors declare that there is no conflict of interest.

\section{References}

1. Pilliar R Blackwell R, Macnab I, Cameron HU. Carbon fiber-reinforced bone cement in orthopedic surgery. J Biomed Mater Res. 1976;10(6):893-906.

2. Hillock R, Howard S. Utility of carbon fiber implants in orthopedic surgery: literature review. Reconstructive Review. 2014;4(1):23-32.

3. Hak DJ, Mauffrey C, Seligson D, et al. Use of carbon-fiber-reinforced composite implants in orthopedic surgery. Orthopedics. 2014;37(12):825-830.

4. Wilson WK, Morris RP, Ward AJ, et al. Torsional Failure of Carbon Fiber Composite Plates Versus Stainless Steel Plates for Comminuted Distal Fibula Fractures. Foot Ankle Int. 2016;37(5):548-553.

5. Hedjazi G. Assessment of processing techniques for Orthopaedic Composites. USA; 2009.
6. Chandramohan D, Marimuthu K. Bio composite materials based on bio polymers and natural fibers-contribution as bone implants. IJAMSAR. 2011;1(1):9-12.

7. Ozdemir MT, Kir MC. Repair of long bone defects with demineralized bone matrix and autogenous bone composite. Indian J Orthop. 2011;45(3):226-230.

8. Wang L, Loh KJ. Wearable carbon nanotube-based fabric sensors for monitoring human physiological performance. Smart Materials and Structures. 2017;26(5):055018.

9. Biercuk M. Carbon nanotube composites for thermal management. Applied physics letters. 2002;80(15):2767-2769.

10. Blasdel NJ. Fabric nanocomposite resistance temperature detector. IEEE Sensors Journal. 2015;15(1):300-306.

11. Liu H, Huaibin Q, Tian J, et al. A promising material for human-friendly functional wearable electronics. Materials Science and Engineering: R: Reports. 2017;112:1-22.

12. Pienkowski DA, Andrews RJ. Polymethylmethacrylate augmented with carbon nanotube, USA; 2003.

13. Arun S, Kanagaraj S. Performance enhancement of epoxy based sandwich composites using multiwalled carbon nanotubes for the application of sockets in trans-femoral amputees. Journal of the mechanical behavior of biomedical materials. 2016;59:1-10.

14. Nan CW. Interface effect on thermal conductivity of carbon nanotube composites. Applied Physics Letters. 2004;85(16):3549-3551.

15. Nan CW, Shi Z, Lin Y. A simple model for thermal conductivity of carbon nanotube-based composites. Chemical Physics Letters. 2003;375(5):666-669.

16. Pötschke P, Bhattacharyya AR, Janke A. Carbon nanotube-filled polycarbonate composites produced by melt mixing and their use in blends with polyethylene. Carbon. 2004;42(5):965-969.

17. Mahdavi M. Effective thermal and mechanical properties of short carbon fiber/natural rubber composites as a function of mechanical loading. Applied Thermal Engineering. 2017;117:8-16.

18. Tian T. Anisotropic thermal property measurement of carbon-fiber/epoxy composite materials. The University of Nebraska-Lincoln, USA; 2011. 